\title{
Prognostic value of CT coronary angiography in diabetic and non-diabetic subjects with suspected CAD: importance of presenting symptoms
}

\author{
Erica Maffei • Sara Seitun - Chiara Martini • Andrea Igoren Guaricci • \\ Giuseppe Tarantini • Niels van Pelt • Annick C. Weustink • Nico R. Mollet • \\ Elena Berti • Roberto Grilli • Giancarlo Messalli • Annachiara Aldrovandi • \\ Filippo Cademartiri
}

Received: 9 March 2010/Revised: 11 October 2010 /Accepted: 10 November 2010 / Published online: 8 December 2010

(C) The Author(s) 2010. This article is published with open access at Springerlink.com

\begin{abstract}
Aim To assess the prognostic relevance of 64-slice computed tomography coronary angiography (CT-CA) and symptoms in diabetics and non-diabetics referred for cardiac evaluation.

Methods We followed 210 patients with diabetes type 2 (DM) and 203 non-diabetic patients referred for CT-CA for ruling out coronary artery disease (CAD). Patients were
\end{abstract}

E. Maffei · S. Seitun · C. Martini · A. I. Guaricci · G. Tarantini

A. Aldrovandi $\cdot$ F. Cademartiri

Department of Radiology and Cardiology,

Azienda Ospedaliero-Universitaria di Parma,

Parma, Italy

\section{G. Tarantini}

Department of Cardiology, University of Padua,

Padua, Italy

N. van Pelt $\cdot$ A. C. Weustink $\cdot$ N. R. Mollet $\cdot$ F. Cademartiri Department of Radiology and Cardiology,

Erasmus Medical Center,

Rotterdam, The Netherlands

E. Berti $\cdot$ R. Grilli

Healthcare and Social Agency,

Regione Emilia-Romagna,

Bologna, Italy

G. Messalli

SDN-IRCCS Foundation,

Naples, Italy

F. Cademartiri $(\bowtie)$

Department of Radiology,

Azienda Ospedaliero-Universitaria - Parma,

43100 Parma, Italy

e-mail: filippocademartiri@hotmail.com without known history of CAD and were divided into four categories on the basis of symptoms at presentation (none, atypical angina, typical angina and dyspnoea). Clinical end points were major cardiac events (MACE): cardiac-related death, non-fatal myocardial infarction, unstable angina and cardiac revascularizations. Cox proportional hazard models, with and without adjustment for risk factors and multiplicative interaction term (obstructive $\mathrm{CAD} \times \mathrm{DM}$ ), were developed to predict outcome.

Results DM patients with dyspnoea or who were asymptomatic showed a higher prevalence of obstructive CAD than non-diabetics $(p \leq 0.01)$. At mean follow-up of 20.4 months, DM patients had worse cardiac event-free survival in comparison with non-DM patients $(90 \%$ vs. $81 \%, p=0.02$ ). In multivariate analysis, CT-CA evidence of obstructive CAD (in DM patients: HR: 6.4; 95\% CI: 2.3-17.5; $p<0.001$; in non-DM patients: HR: 7.4 ; $95 \%$ CI: $2.1-26.7$; $p=0.002$ ) and the presence of typical angina (in DM patients: HR: 2.9 ; 95\% CI: $1.3-6.3$; $\mathrm{p}=0.007$; in non-DM patients: HR: 2.7 ; 95\% CI: $1.1-7.1 ; p=0.03$ ) were independent predictors of MACE in both groups. Furthermore, other independent outcome predictors included dyspnoea (HR: $3.8 ; 95 \%$ CI: $1.7-8.5 ; \mathrm{p}=0.001$ ), the number of segments with any CAD (HR: $1.1 ; 95 \%$ CI: 1.001-1.2; $p=0.04)$ in DM patients and coronary calcium score $>100$ in non-DM patients (HR: 5.6; 95\% CI: 1.4-21.5; $p=0.01$ ). In Cox regression analysis of the overall population, interaction term obstructive $\mathrm{CAD} \times \mathrm{DM}$ resulted in non-significance.

Conclusions Among DM patients, dyspnoea carried a high event risk with a MACE rate four times higher. CT-CA findings were strongly predictive of outcome and proved valuable for further risk stratification. 
Keywords Prognostic value · CT coronary angiography . Diabetes mellitus $\cdot$ Symptoms

\section{Introduction}

Type 2 diabetes mellitus (DM) is an increasing health care problem [1]. The most common cause of death in European adults with DM is coronary artery disease (CAD) [2]. Several studies have demonstrated that in patients with DM, the risk of major cardiovascular events is two to four times higher than that among non-DM patients [3].

The role of non-invasive coronary imaging in diabetic patients, who typically have a high prevalence of obstructive CAD $[4,5]$, is not to simply document the presence of coronary atherosclerosis, but to identify those patients with more extensive disease. In these patients, further functional testing could assess the haemodynamic significance of the demonstrated CAD. Despite the well-validated negative predictive value of stress imaging techniques after a normal stress test $[6,7]$, diabetic patients have a more than two-fold increased likelihood of myocardial infarction or cardiac death compared with non-diabetic patients [8]. Therefore, an extended risk assessment is important in this population in order to identify those patients at particular risk of future cardiac events and outline goals for the long-term management strategy. It is generally accepted that the character of the symptoms in patients with suspected angina is central to clinical diagnosis and risk assessment [9-11]. A difficulty in the assessment of patients with DM is that symptoms of myocardial ischaemia are often absent or atypical (shortness of breath) [12].

Recently, multislice computed tomography coronary angiography (MSCT-CA) has emerged as a powerful tool for ruling out $\mathrm{CAD}$, especially in patients with an intermediate likelihood of $\mathrm{CAD}$ or when testing for ischaemia is equivocal [13, 14]. Previous studies of MSCT-CA have focused primarily on its diagnostic accuracy for the detection of obstructive CAD in comparison with invasive coronary angiography and demonstrated a high sensitivity and negative predictive value close to $100 \%$ [15]. The ability to detect not only coronary stenosis but also the non-obstructive coronary atherosclerotic plaque in a non-invasive fashion makes MSCT-CA imaging a potentially valuable tool for risk stratification. Over the past 3 years, the clinical value of MSCT-CA to determine prognosis has begun to be investigated [16-22]. However, most of these studies have focused on patients with either a suspected or documented history of CAD [16-19].

The goal of the present study was to evaluate the prognostic implications of MSCT-CA findings as well as presenting symptoms in diabetic patients with suspected CAD compared with non-diabetic individuals who un- derwent MSCT-CA with the aim of ruling out CAD. Furthermore, we sought to identify how the MSCT-CA findings can be combined with symptom evaluation to further refine the prognostic power of MSCT-CA and, thus, allow for the more effective identification of patients at relatively high (or low) risk of future cardiac events.

\section{Materials and methods}

Study group The study group consisted of 433 consecutive eligible patients who were referred to our institution from January 2005 to December 2005 for further evaluation of suspected CAD on the basis of symptoms, elevated risk profile or abnormal diagnostic test results. All patients were without known history of CAD [defined as chart documented $>30 \%$ luminal narrowing in any vessel on previous $\mathrm{CAG}$, acute myocardial infarction (MI), a previous history of MI, coronary artery bypass graft surgery or percutaneous coronary intervention]. The study was a single-centre prospective observational study. In all patients MSCT-CA was performed on the basis of symptoms and/or elevated risk profile in addition to standard clinical diagnostic workup involving exercise electrocardiogram, myocardial scintigraphy and echocardiography.

From the 433 patients who had an MSCT-CA, 20 (5\%) were lost to follow-up, resulting in a final study cohort of 413 individuals. Of these 413 patients, 210 had DM, while 203 were without DM. The enrolment was performed consecutively in each group (DM and non-DM). At the baseline examination, a standardised data collection form was filled out by the duty physician (F.C.; E.M.). Baseline demographic variables, CAD risk factors and clinical signs were collected.

Diabetes was defined as a fasting plasma glucose level of $\geq 126 \mathrm{mg} / \mathrm{dl}$ treated currently with diet intervention, oral glucose-lowering agents or insulin [23]. The following risk factors were acquired for all subjects at the baseline examination: systemic hypertension (defined as blood pressure of $\geq 140 / 90 \mathrm{mmHg}$ or the use of antihypertensive medication) [24]; hypercholesterolaemia (defined as a total cholesterol level of $\geq 200 \mathrm{mg} / \mathrm{dl}$ or treatment with lipid-lowering drugs) [25]; obesity (body mass index of $\geq 30 \mathrm{~kg} / \mathrm{m}^{2}$ ) [26]; positive family history of CAD (defined as the presence of CAD in a first-degree female $[<65$ years] or male $[<55$ years] relative [27]); and smoking (defined as previous or current smoking).

Patients were divided into four categories according to their self-reported symptoms of chest pain and dyspnoea at the time of testing. Typical angina was defined as chest pain that was substernal, occurring during stress and resolving within $10 \mathrm{~min}$ after rest or the receipt of nitroglycerin, 
according to the criteria of Diamond [9]. Patients with chest pain symptoms that did not fall into this typical category were further classified as having atypical angina. Among patients without chest discomfort, patients who experienced shortness of breath were classified as having dyspnoea; the remainder were classified as asymptomatic. Dyspnoea was not coded in patients with chest pain.

Pre-test likelihood of CAD was determined in every patient according to a modification of the DiamondForrester algorithm, as published by Morise et al. [28]. These patients were further categorised into low probability (score 0-8), intermediate probability (score 9-15) and high probability (score $>15$ ).

Inclusion criteria for the imaging were regular heart rate (spontaneous or $\beta$-blocker-induced) of less than 70 beats per minute (bpm) and the ability to hold a breath for at least $12 \mathrm{~s}$. Exclusion criteria for the imaging were a high heart rate $(>70 \mathrm{bpm})$, previous allergic reaction to iodine contrast medium, renal insufficiency (creatinine clearance $<60 \mathrm{ml} / \mathrm{min}$ ), pregnancy, respiratory impairment and unstable clinical status.

The study was approved by the institutional review board, and informed consent was obtained from all participating patients before testing.

MSCT protocol All examinations were performed with a 64-slice CT scanner (Sensation 64, Siemens, Forchheim, Germany) as described elsewhere [15]. First a non-contrastenhanced, prospectively ECG-triggered CT was performed. This CT was used to calculate the Agatston calcium score (CACS). Afterwards we performed MSCT-CA, after an intravenous injection of a bolus $(80-100 \mathrm{ml}$ at $4-6 \mathrm{ml} / \mathrm{s})$ of non-ionic iodinated contrast agent (iomeprol $400 \mathrm{mg} / \mathrm{ml}$, Iomeron, Bracco, Italy) followed by a saline chaser $(50 \mathrm{ml}$ at $4-6 \mathrm{ml} / \mathrm{s}$ ). If the heart rate was $>65 \mathrm{bpm}$, additional intravenous beta-blockers (atenolol 5-10 mg) were provided when tolerated. In 308 patients (75\%) sublingual nitroglycerin $(0.3 \mathrm{mg})$ was also administered immediately before the examination to optimise visualisation of small coronary vessels.

Tube current modulation was not used; the mean radiation exposure using this imaging protocol was 685 mGy (range 515-1,052 mGy), corresponding to an effective dose of $11.6 \mathrm{mSv}$ (range $8.7-17.9 \mathrm{mSv}$ ).

MSCT data analysis With dedicated software (CaScore, Siemens, Germany), an overall CACS was recorded for each patient based on the scoring algorithm of Agatston et al. [29], where coronary artery calcium was identified as a dense area greater than $1 \mathrm{~mm}^{2}$ in the coronary artery exceeding the threshold of 130 Hounsfield units. All MSCT-CA investigations were evaluated by two experienced observers who were unaware of the clinical history of the patients, using a standard approach to analysis. In the case of disagreement, a joint reading was performed and a consensus decision was reached. All 16 coronary segments as established in the American Heart Association classification [30] were considered in the analysis. First, each segment was rated as interpretable or not interpretable. Thereafter, all interpretable segments were evaluated for the presence of any atherosclerotic plaque [31] using axial images and curved multiplanar reconstructions. Subsequently, the lesion was classified visually as obstructive $(>50 \%$ luminal narrowing) or non-obstructive $(\leq 50 \%$ luminal narrowing). For every patient, the number of diseased segments with obstructive and non-obstructive CAD was recorded. Finally, a total plaque score (out of 16) including all affected segments was calculated in each patient.

Follow-up All patients were followed up for a minimum of 6 months after the MSCT-CA examination. One of the study investigators (S.S.; E.M.; F.C.) performed a telephone interview with each patient, with his or her direct relative or with the referring physician to ascertain follow-up information and screen for occurrence of clinical events or coronary revascularization procedures, any change in clinical status and hospital admission. To confirm the obtained information, electronic hospital records of all patients were carefully screened for the occurrence of clinical events.

The principal study end point was the composite of major adverse cardiac events (MACE), which included the need for elective revascularization (including during the initial assessment) and hard cardiac events: cardiac death, non-fatal MI and unstable angina requiring hospitalization (UA). Deaths that could not be classified were considered cardiac-related. MI was defined based on criteria of typical chest pain, elevated cardiac enzyme levels and typical changes on the electrocardiogram [32]. The decision regarding the need for coronary revascularization and the method of revascularization (coronary artery bypass grafting or a percutaneous coronary intervention) was made by the referring cardiologist on an individual patient basis.

Statistical analysis Continuous variables are expressed as mean values $( \pm \mathrm{SD})$ or median and interquartile range $(25 \%$ and $75 \%$ percentiles), where appropriate. Differences between groups were compared using the Student's $t$ and chi-squared tests, as appropriate.

Cumulative event rates of the composite MACE (cardiac death, non-fatal MI, UA and the need for revascularization) were estimated using the Kaplan-Meier method and compared using the log-rank test. A parallel survival model was constructed in which patients with early coronary revascularization ( $<60$ days after the MSCT-CA examination) were excluded from the analysis. Patients undergoing coronary revascularization were censored at the time of the 
procedure. In the case of multiple events for the same person only the first event was counted. Survival times of patients still alive or disease-free were censored with the median follow-up period.

The association of selected variables with MACE was assessed using Cox's proportional hazards survival model involving univariate and forward stepwise multivariate procedures. A significance level of 0.05 was required for an MSCT-CA variable to be included in the multivariate model, whereas a level of 0.1 was the cutoff value for exclusion. Multivariate analysis was corrected for the baseline characteristics with $p \leq 0.1$ in the univariate analysis. Hazard ratios with the corresponding 95\% confidence intervals (CI) were estimated. Potential interaction (effect modification) between obstructive CAD and diabetes was evaluated by adding a multiplicative interaction term (obstructive CAD $\times$ diabetes) to the Cox model.

The predictive value of CACS was assessed using binary cutoff values (CACS $\leq 100, \mathrm{CACS}>100, \mathrm{CACS}>400)$. The incremental value of MSCT-CA was determined by calculating the change in global $\chi^{2}$ with respect to prognosis (Cox model) after adding the MSCT-CA variables to the pre-imaging information (Morise score) and significant calcium score $(\mathrm{CACS}>100)$. Annualized event rates were expressed as a proportion of the number of patients having MACE divided by the number of patientyears' follow-up.

Receiver-operating characteristic (ROC) analysis was performed to calculate the area under the curve (AUC) to determine whether the total plaque score provided incremental value in predicting MACE over and above that provided by the pre-test likelihood prediction model according to Morise.

Statistical analysis was performed with SPSS (version 12.0, SPSS, Chicago, IL) and MedCalc (version 9.3.0.0., MedCalc Software, Mariakerke, Belgium) software. Significance was set at $p<0.05$.

\section{Results}

All 413 patients included in the study underwent MSCT-CA without complications. The clinical and demographic characteristics of the patients are shown in Table 1. Overall, 61 (15\%) patients had a low, $302(73 \%)$ had an intermediate and $50(12 \%)$ had a high pre-test likelihood of CAD. The two groups studied were comparable with regard to the baseline characteristics, but differed significantly with regard to BMI, smoking history and pre-test likelihood of CAD (low and high categories). Most DM and non-DM patients were symptomatic ( $66 \%$ vs. $72 \%$, respectively, $p$ n.s.), with atypical angina the more frequent presenting symptom ( $36 \%$ vs. $38 \%$, respectively, $p$ n.s.).
MSCT-CA findings A total of $92(1 \%)$ coronary segments were considered to be of non-diagnostic quality ( $\mathrm{n}=80$ with motion artefacts due to elevated heart rate, $n=12$ with extensive calcification) and were excluded from evaluation. Total plaque burden was therefore evaluated in 6,227 segments. As shown in Table 2, patients with DM had twice the prevalence of obstructive CAD compared with non-diabetic patients $(36 \%$ vs. $17 \%$, respectively, $p<$ 0.0001 ), and a lower prevalence of normal coronary arteries (28\% vs. $37 \%$, respectively) and non-obstructive CAD ( $36 \%$ vs. $46 \%$, respectively), although these differences were non-significant. The total Agatston calcium score, which reflects plaque burden, was higher in diabetic patients than in normal patients (351 vs. 146, respectively, $p<0.001)$. Furthermore, diabetic patients showed a higher prevalence of CACS $>400$ ( $24 \%$ vs. $11 \%$, respectively, $p=$ 0.001 ) and a higher average number of diseased coronary segments ( 4.5 vs. 2.8 , respectively, $p<0.0001$ ), with either obstructive $(0.9$ vs. $0.3, p<0.0001)$ or non-obstructive $(3.7$ vs. $2.5, p<0.001)$ CAD.

Prevalence of CAD on MSCT-CA in DM and non-DM patients stratified for the type of presenting symptom is provided in Fig. 1. In both DM and non-DM patients, the highest prevalence of obstructive CAD was documented in patients with typical angina $(57.7 \%$ vs. $56.5 \%$, respectively, $p=$ n.s.).

There was a higher prevalence of obstructive CAD in DM patients than in non-DM patients in all symptom categories, although this difference was only significant in asymptomatic patients $(37.5 \%$ vs. $10.7 \%$, respectively, $p=0.001)$ and patients with dyspnoea $(35.1 \%$ vs. $10.6 \%$, respectively, $p=0.01$ ).

Follow-up results During a mean follow-up period of 20.4 \pm 5.2 months (median: 580 days, interquartile range: 420-686 days), 37 MACE occurred in diabetic patients compared with 20 in non-diabetic patients $(18 \%$ vs. $10 \%$, respectively, $p=0.03$ ). Excluding revascularization, a total of ten hard cardiac events were observed, eight hard events (fatal acute MI, $\mathrm{n}=2$; non-fatal acute MI, $\mathrm{n}=3$; UA requiring hospitalization, $n=3$ ) among DM patients (4\%) and two hard events (non-fatal acute MI, $\mathrm{n}=2$ ) among nonDM patients $(1 \%)$.

Of the 37 diabetic patients suffering cardiac events, 31 $(84 \%)$ had obstructive disease, while $6(16 \%)$ had nonobstructive disease $(p<0.0001)$. Conversely, the 20 nondiabetic patients suffering cardiac events comprised 16 $(80 \%)$ with obstructive disease and $4(20 \%$ with nonobstructive disease $(p<0.0001)$.

A total of $33(16 \%)$ diabetic and $18(9 \%)$ non-diabetic patients $(p=0.04)$ underwent coronary revascularization (10 surgical and 41 percutaneous coronary interventions). Four revascularizations were performed after the occur- 
Table 1 Baseline characteristics of the overall population and of diabetics compared with non-diabetics

\begin{tabular}{|c|c|c|c|c|}
\hline & Overall population $(n=413)$ & Diabetics $(n=210)$ & Non-diabetics $(n=203)$ & $p$ Value* \\
\hline \multicolumn{5}{|l|}{ Clinical characteristics } \\
\hline Age (years; mean [SD]) & $61(11)$ & $60(10)$ & $62(11)$ & 0.06 \\
\hline Male gender $(\%)$ & $262(63)$ & $133(63)$ & $129(63)$ & 0.95 \\
\hline BMI $\left(\mathrm{kg} / \mathrm{m}^{2} ;\right.$ mean $\left.[\mathrm{SD}]\right)$ & $27(2)$ & $28(4)$ & $26(3)$ & $<0.0001$ \\
\hline Mean heart rate (bpm; mean [SD]) & $61(10)$ & $62(10)$ & $61(10)$ & 0.31 \\
\hline Follow-up (months; mean [SD]) & $20(6)$ & $20(8)$ & $21(3)$ & 0.16 \\
\hline \multicolumn{5}{|l|}{ Risk factors } \\
\hline N. of risk factors (mean [SD]) & $2.3(1.2)$ & $2.3(1.2)$ & $2.2(1.1)$ & 0.38 \\
\hline Hypertension & $272(66)$ & $144(69)$ & $128(63)$ & 0.28 \\
\hline Hypercholesterolaemia (\%) & $200(48)$ & $97(46)$ & $103(51)$ & 0.41 \\
\hline Obesity $\left(\mathrm{BMI} \geq 30 \mathrm{~kg} / \mathrm{m}^{2}\right)(\%)$ & $117(28)$ & $78(37)$ & 39 (19) & 0.0001 \\
\hline Current smoking (\%) & $144(35)$ & $63(30)$ & $81(40)$ & 0.04 \\
\hline Family history of CAD (\%) & $200(48)$ & $105(50)$ & $95(47)$ & 0.58 \\
\hline \multicolumn{5}{|l|}{ Symptoms } \\
\hline Asymptomatic (\%) & $128(31)$ & $72(34)$ & $56(28)$ & 0.17 \\
\hline Typical angina pectoris $(\%)$ & $49(12)$ & $26(12)$ & $23(11)$ & 0.86 \\
\hline Atypical angina pectoris (\%) & $152(37)$ & $75(36)$ & $77(38)$ & 0.71 \\
\hline Dyspnoea (\%) & $84(20)$ & $37(18)$ & $47(23)$ & 0.20 \\
\hline \multicolumn{5}{|l|}{ Pre-test likelihood of $\mathrm{CAD}^{\#}$} \\
\hline Low $(\%)$ & $61(15)$ & $19(9)$ & $42(21)$ & 0.001 \\
\hline Intermediate $(\%)$ & $302(73)$ & $148(71)$ & $154(76)$ & 0.26 \\
\hline High $(\%)$ & $50(12)$ & $43(20)$ & $7(3)$ & $<0.0001$ \\
\hline
\end{tabular}

Data are presented as mean (standard deviation) or number (percentage). $\mathrm{CAD}=$ coronary artery disease; $\mathrm{BMI}=$ body mass index. ${ }^{\#}$ According to the scoring method of Morise. *Comparison between diabetics and non-diabetics

rence of a hard cardiac event in four DM patients. Thirtyone (61\%) patients underwent revascularization because of single-vessel disease. Twenty-nine (57\%) patients underwent revascularization within 2 months of the MSCT-CA examination.

Annualized MACE rates in DM and non-DM patients stratified with regard to the type of presenting symptoms are provided in Table 3. In both DM and non-DM patients, the highest annual MACE rate was documented in patients with typical angina $(26.3 \%$ vs. $23.2 \%$, respectively, $p=$ n.s. $)$. Furthermore, in both DM and non-DM patients, those with typical angina had significantly more MACE than asymptomatic patients $(p<0.01)$ or those with atypical angina $(p<0.05)$. Significant difference was also observed among DM patients with dyspnoea and asymptomatic DM patients ( $20.2 \%$ vs. $5.5 \%$, respectively, $p=0.04)$.

Survival analysis As shown in Fig. 2, DM patients had a worse prognosis than non-DM patients $(18.7 \%$ vs. $9.9 \%$, respectively, $p=0.02$ ). In both DM and non-DM patients a higher MACE rate occurred in patients with obstructive CAD (42.7\% vs. $45.7 \%$, respectively) compared with patients with non-obstructive CAD $(9.8 \%$ vs. $4.3 \%)$ or normal coronary arteries $(0 \%$ vs. $0 \%)(p<0.0001)$ (Fig. 3$)$.
After stratification in terms of presenting symptoms (Fig. 4), the MACE rate was higher in both DM and nonDM patients with typical angina compared with the other clinically relevant categories $(p<0.0001)$. Notably, among DM patients, the MACE rate was significantly higher in patients with dyspnoea than in asymptomatic patients ( $27.9 \%$ vs. $8.7 \%$, respectively, $p=0.005)$, and after comparing patients with dyspnoea with patients with atypical angina the difference was nearly significant $(27.9 \%$ vs. $13.6 \%$, respectively, $p=0.05$ ). Moreover, among patients with dyspnoea, the MACE rate was higher in DM patients than in non-DM patients $(27.9 \%$ vs. $8.5 \%$, respectively, $p=0.02$ ).

After excluding early revascularizations $(<60$ days after MSCT-CA examination, $n=29$ ), survival analysis of MACE $(n=28)$ demonstrated that the event rate remained higher among DM patients than among non-DM patients (11.6\% vs. $3.0 \%$, respectively, $p=0.001)$. Moreover, the presence of obstructive CAD and typical angina remained significantly associated with outcome in both DM and non-DM patients $(p<0.001)$.

Outcome prediction Univariate and multivariate predictors of MACE for all patients and for DM and non-DM patients 
Table 2 Multislice computed tomography coronary angiography findings of the overall population and of diabetics compared with non-diabetics

Data are presented as mean (standard deviation) or number (percentage). $\mathrm{CAD}=$ coronary artery disease; $\mathrm{LM}=$ left main coronary artery; $\mathrm{LAD}=$ left anterior descending coronary artery; $\mathrm{RCA}=$ right coronary artery; $\mathrm{CXA}=$ left circumflex coronary artery; CACS, coronary artery calcium score.

*Comparison between diabetics and non-diabetics

\begin{tabular}{|c|c|c|c|c|}
\hline & $\begin{array}{l}\text { Overall population } \\
(\mathrm{n}=413)\end{array}$ & $\begin{array}{l}\text { Diabetics } \\
(\mathrm{n}=210)\end{array}$ & $\begin{array}{l}\text { Non-diabetics } \\
(\mathrm{n}=203)\end{array}$ & $p$ Value* \\
\hline \multicolumn{5}{|l|}{ Patients } \\
\hline Absence of CAD (\%) & $134(32)$ & $59(28)$ & $75(37)$ & 0.07 \\
\hline Non-obstructive CAD (\%) & $169(41)$ & $76(36)$ & $93(46)$ & 0.06 \\
\hline Obstructive CAD (\%) & $110(27)$ & $75(36)$ & $35(17)$ & $<0.0001$ \\
\hline \multicolumn{5}{|l|}{ Obstructive CAD in: } \\
\hline $\mathrm{LM} / \mathrm{LAD}$ & $76(18)$ & $54(26)$ & $22(11)$ & $<0.001$ \\
\hline $\mathrm{RCA}$ & $43(10)$ & $32(15)$ & $11(5)$ & 0.001 \\
\hline CXA & $37(9)$ & $25(12)$ & $12(6)$ & 0.05 \\
\hline Single-vessel disease (\%) & $72(17)$ & $46(22)$ & $26(13)$ & 0.02 \\
\hline Multivessel disease $(\%)$ & $38(9)$ & $29(14)$ & $9(4)$ & 0.002 \\
\hline Total Agatston score & $250.7(599.7)$ & $351.6(743.4)$ & $146.2(374.5)$ & $<0.001$ \\
\hline $\mathrm{CACS} \leq 100$ & $277(67)$ & $132(63)$ & $145(71)$ & 0.08 \\
\hline $\mathrm{CACS}>100$ & $136(33)$ & $78(37)$ & $58(29)$ & 0.08 \\
\hline $\mathrm{CACS}>400$ & $73(18)$ & $50(24)$ & $23(11)$ & 0.001 \\
\hline \multicolumn{5}{|l|}{ Segments } \\
\hline No. of diseased segments & $3.7(4.2)$ & $4.5(4.6)$ & $2.8(3.5)$ & $<0.0001$ \\
\hline \multicolumn{5}{|l|}{ No. of segments with } \\
\hline Non-obstructive plaque & $3.1(3.7)$ & $3.7(4.0)$ & $2.5(3.2)$ & $<0.001$ \\
\hline Obstructive plaque & $0.6(1.4)$ & $0.9(1.8)$ & $0.3(0.8)$ & $<0.0001$ \\
\hline
\end{tabular}

are reported in Tables 4 and 5. Typical angina, dyspnoea, CACS $>100$ and obstructive CAD were independently associated with MACE in all patients $(n=413)$ (Table 4). The interaction term obstructive $\mathrm{CAD} \times$ diabetes was highly significant $(p<0.0001)$ at univariate analysis and suggested that obstructive $\mathrm{CAD}$ and $\mathrm{DM}$ were synergistic for predicting survival. However, when incorporated in the multivariate Cox proportional hazards regression analysis, the interaction term was not significant.

After adjusting for baseline risk factors and presenting symptoms obtained before MSCT-CA, the presence of obstructive CAD remained the strongest predictor of events for both DM and non-DM patients, thus yielding incremental value over pre-imaging data (Table 5). Furthermore, typical angina was a multivariate prognostic indicator for both groups $(p<0.05)$, while the presence of dyspnoea was also a strong independent predictor only for DM patients $(p=0.001)$, together with increasing age $(p=0.03)$ and the number of segments with any CAD $(p=0.04)$. The presence of CACS $>100$ remained a significant outcome predictor only for non-diabetics $(p=0.01)$.

The incremental value of coronary plaque content over traditional risk assessment was evaluated by ROC analysis (Fig. 5). The AUC for the total plaque score predicting MACE was significant in all patients $(0.83$, CI $0.78-0.87$, $p<0.0001)$ and in both non-diabetics (0.84, CI 0.78-0.90, $p<0.0001)$ and diabetics (0.81, CI 0.74-0.88, $p<0.0001$ ). Similarly, the AUC for pre-test likelihood predicting MACE
Fig. 1 Clustered bar graph showing the association between symptom categories and the prevalence of normal/ non-obstructive $\mathrm{CAD}$ and obstructive CAD in DM and non-DM patients.

$\mathrm{CAD}=$ coronary artery disease; DM: diabetes mellitus

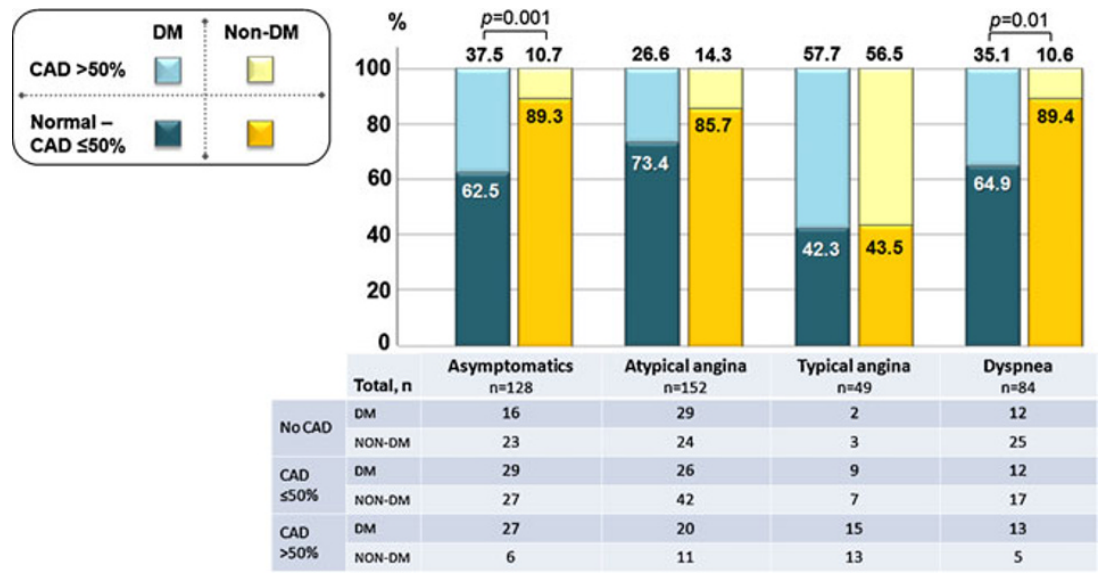



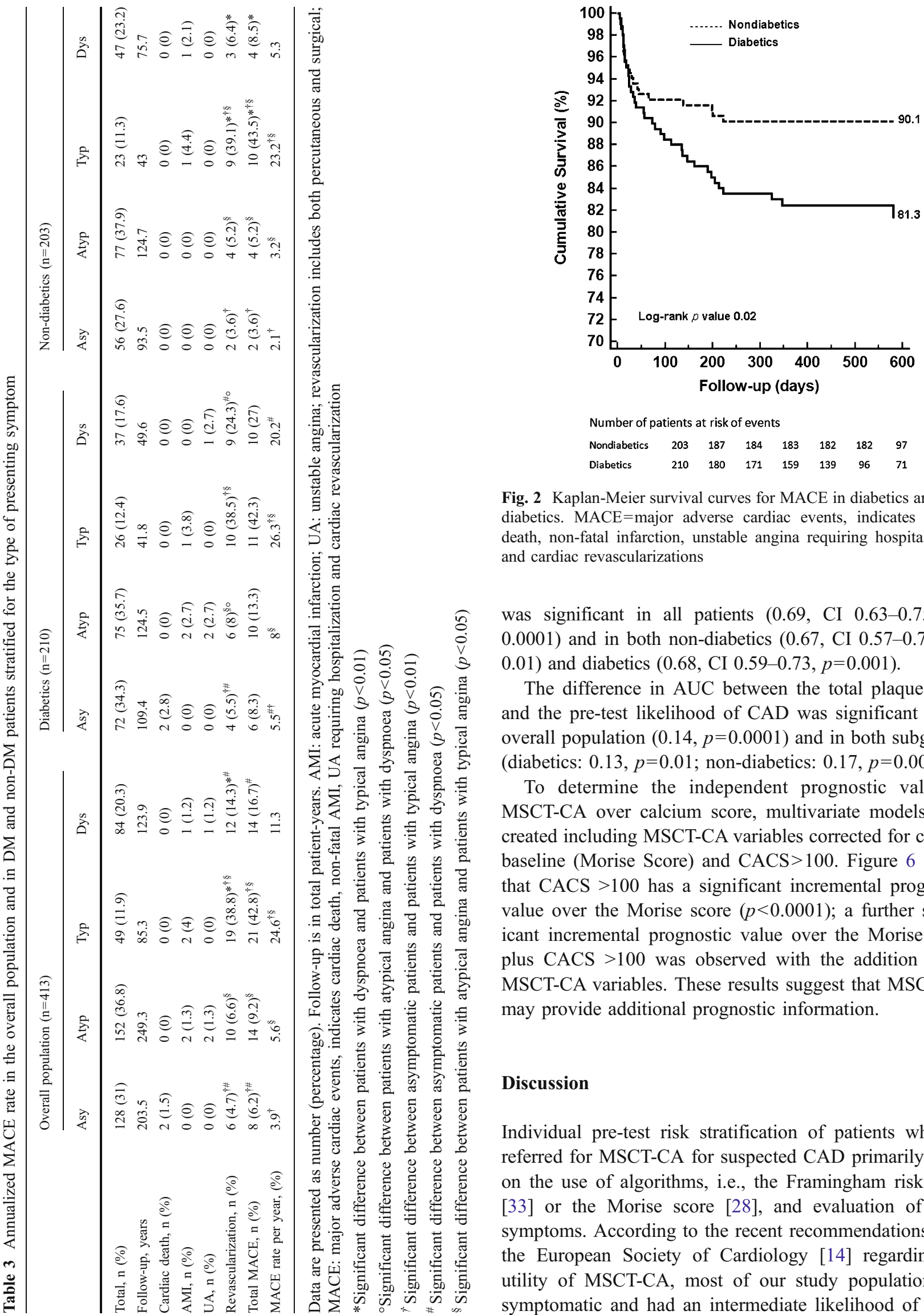

Fig. 2 Kaplan-Meier survival curves for MACE in diabetics and nondiabetics. $\mathrm{MACE}=$ major adverse cardiac events, indicates cardiac death, non-fatal infarction, unstable angina requiring hospitalization and cardiac revascularizations

was significant in all patients $(0.69$, CI $0.63-0.75, p<$ $0.0001)$ and in both non-diabetics $(0.67$, CI $0.57-0.76, p=$ $0.01)$ and diabetics $(0.68$, CI $0.59-0.73, p=0.001)$.

The difference in AUC between the total plaque score and the pre-test likelihood of CAD was significant in the overall population $(0.14, p=0.0001)$ and in both subgroups (diabetics: $0.13, p=0.01$; non-diabetics: $0.17, p=0.004$ ).

To determine the independent prognostic value of MSCT-CA over calcium score, multivariate models were created including MSCT-CA variables corrected for clinical baseline (Morise Score) and CACS $>100$. Figure 6 shows that CACS $>100$ has a significant incremental prognostic value over the Morise score $(p<0.0001)$; a further significant incremental prognostic value over the Morise score plus CACS $>100$ was observed with the addition of all MSCT-CA variables. These results suggest that MSCT-CA may provide additional prognostic information.

\section{Discussion}

Individual pre-test risk stratification of patients who are referred for MSCT-CA for suspected CAD primarily relies on the use of algorithms, i.e., the Framingham risk score [33] or the Morise score [28], and evaluation of chest symptoms. According to the recent recommendations from the European Society of Cardiology [14] regarding the utility of MSCT-CA, most of our study population was symptomatic and had an intermediate likelihood of CAD. 
Fig. 3 Kaplan-Meier survival curves for MACE in diabetics and non-diabetics stratified for the severity of CAD on MSCT-CA: Absence of plaques, non-obstructive disease and obstructive disease. $\mathrm{MACE}=$ major adverse cardiac events, indicates cardiac death, non-fatal infarction, unstable angina requiring hospitalization and cardiac revascularizations. $\mathrm{CAD}=$ coronary artery disease; MSCT-CA $=$ multislice computed tomography coronary angiography
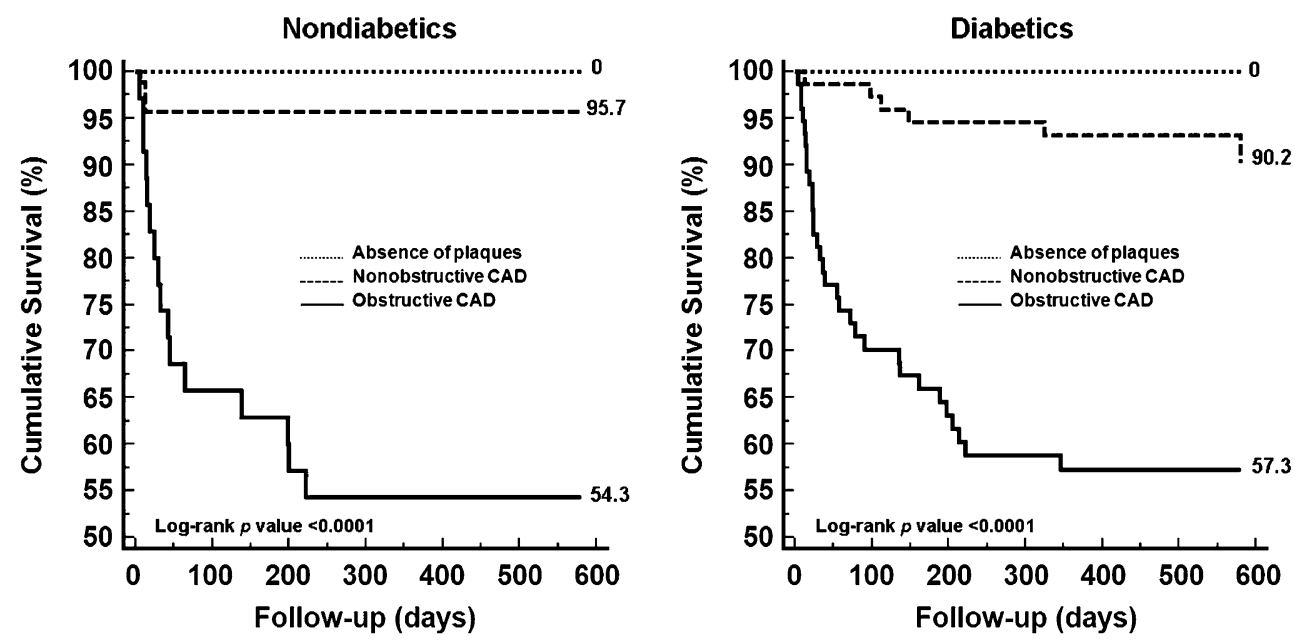

$\begin{array}{llllllll}\text { Number of patients at risk of events } & & & & & \\ \text { Absence of plaques } & 59 & 59 & 58 & 54 & 44 & 25 & 21 \\ \text { Nonobstructive CAD } & 76 & 71 & 69 & 65 & 60 & 47 & 32 \\ \text { Obstructive CAD } & 75 & 50 & 44 & 40 & 35 & 24 & 18\end{array}$
Although there was a higher prevalence of smoking among non-diabetics, diabetic patients had a greater overall baseline risk factor profile, as demonstrated by a higher mean BMI and prevalence of obesity. Furthermore, patients with DM had a larger plaque burden as $\mathrm{CAD}$ tended to be more severe and extensive, resulting in multivessel disease being more frequently diagnosed. These findings have a pathological basis, as demonstrated by previous autopsy study in which, among individuals without clinical CAD, DM was associated with a global coronary disease burden and a prevalence of high-grade atherosclerosis similar to that observed among non-diabetic persons with clinical CAD [4].

In our study, after stratification in terms of presenting symptoms, DM and non-DM patients with typical angina had the highest prevalence of obstructive CAD compared with patients with other clinical presentations. This finding is in agreement with the work by Diamond and Forrester [34], in which patients with typical angina had a higher likelihood of angiographic CAD than patients with atypical or non-anginal chest pain.

Moreover, in our study DM patients more frequently underwent coronary revascularization and fared worse than non-diabetic patients.

On multivariate survival analysis, the presence of obstructive CAD was independently associated with outcome in both DM and non-DM patients. Interestingly, the diabetic state alone was not a significant independent predictor for vascular events in Cox regression analysis of

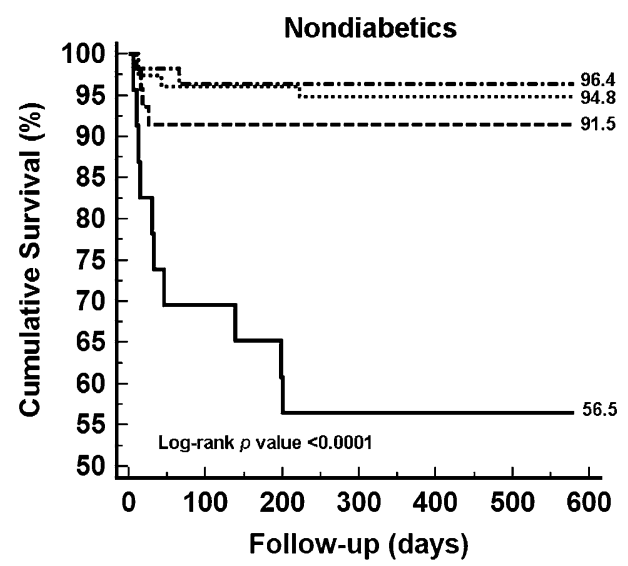

$\begin{array}{llllllll}\text { Number of patients at risk of events } & & & & & \\ \text { Asymptomatics } & 56 & 54 & 54 & 54 & 54 & 54 & 34 \\ \text { Atypical angina } & 77 & 74 & 74 & 73 & 72 & 72 & 31 \\ \text { Dyspnea } & 47 & 43 & 43 & 43 & 43 & 43 & 24 \\ \text { Typical angina } & 23 & 16 & 13 & 13 & 13 & 13 & 8\end{array}$

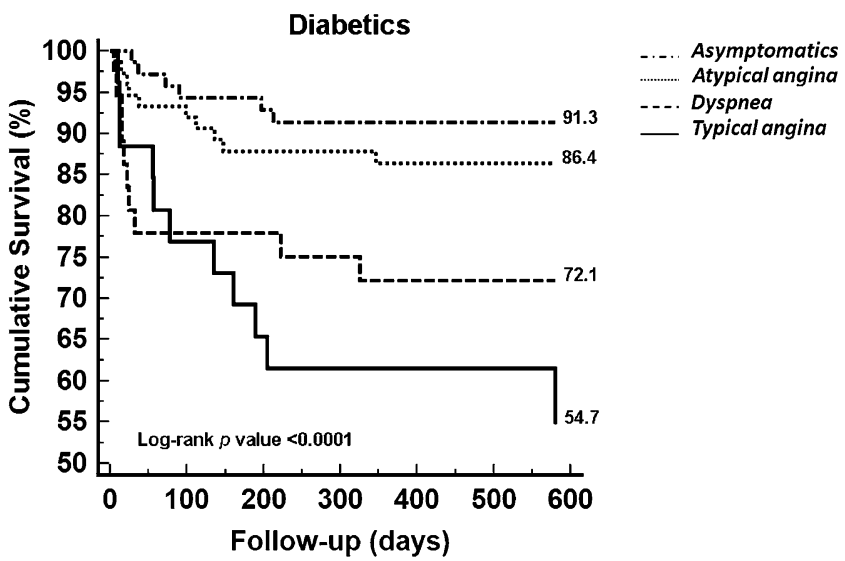

$\begin{array}{llllllll}\text { Number of patients at risk of events } & & & & & \\ \text { Asymptomatics } & 72 & 65 & 63 & 54 & 49 & 32 & 26 \\ \text { Atypical angina } & 75 & 68 & 64 & 63 & 57 & 41 & 30 \\ \text { Dyspnea } & 37 & 27 & 27 & 26 & 20 & 12 & 6 \\ \text { Typical angina } & 26 & 20 & 17 & 16 & 13 & 11 & 9\end{array}$

Fig. 4 Kaplan-Meier survival curves for MACE in diabetics and nondiabetics stratified for the type of presenting symptom. MACE $=$ major adverse cardiac events, indicates cardiac death, non-fatal infarction, unstable angina requiring hospitalization and cardiac revascularizations. $\mathrm{CAD}=$ coronary artery disease; $\mathrm{MSCT}-\mathrm{CA}=$ multislice computed tomography coronary angiography 
Table 4 Univariate and multivariate predictors of total cardiac events in all patients
$\mathrm{CAD}=$ coronary artery disease; $\mathrm{HR}=$ hazard ratio; CACS: Coronary Artery Calcium Score; $\mathrm{DM}=$ diabetes mellitus; $\mathrm{CI}=$ confidence interval

\begin{tabular}{|c|c|c|c|c|}
\hline & \multicolumn{4}{|l|}{ All patients $(n=413)$} \\
\hline & \multicolumn{2}{|l|}{ Univariate analysis } & \multicolumn{2}{|c|}{ Multivariate analysis } \\
\hline & HR $(95 \% \mathrm{CI})$ & $p$ Value & HR $(95 \% \mathrm{CI})$ & $p$ Value \\
\hline \multicolumn{5}{|l|}{ Clinical characteristics } \\
\hline Age ( $>65$ years) & $2.80(1.64-4.79)$ & $<0.001$ & & \\
\hline Male gender & $1.35(0.77-2.38)$ & 0.29 & & \\
\hline $\mathrm{DM}$ & $1.82(1.06-3.14)$ & 0.03 & & \\
\hline Hypertension & $2.02(1.07-3.80)$ & 0.03 & & \\
\hline Family history & $1.63(0.96-2.77)$ & 0.07 & & \\
\hline Smoking & $1.84(1.10-3.09)$ & 0.02 & & \\
\hline Hypercholesterolaemia & $1.76(1.03-2.98)$ & 0.04 & & \\
\hline Obesity & $0.88(0.49-1.59)$ & 0.68 & & \\
\hline \multicolumn{5}{|l|}{ Symptoms } \\
\hline Asymptomatic & $0.34(0.16-0.71)$ & 0.004 & & \\
\hline Typical angina pectoris & $5.05(2.95-8.63)$ & $<0.0001$ & $2.70(1.46-4.97)$ & 0.001 \\
\hline Atypical angina pectoris & $0.53(0.29-0.97)$ & 0.04 & & \\
\hline Dyspnoea & $1.62(0.91-2.88)$ & 0.10 & $3.08(1.55-6.09)$ & 0.001 \\
\hline \multicolumn{5}{|l|}{ MSCT characteristics } \\
\hline CACS $>100$ & $11.39(5.7-22.6)$ & $<0.0001$ & $3.5(1.62-7.57)$ & 0.001 \\
\hline CACS $>400$ & $6.11(3.6-10.3)$ & $<0.0001$ & & \\
\hline Absence of plaques & $0.031(0.006-0.17)$ & $<0.001$ & & \\
\hline Non-obstructive CAD & $0.28(0.14-0.56)$ & $<0.001$ & & \\
\hline Obstructive CAD & $16.7(8.46-33.1)$ & $<0.0001$ & $8.9(4.0-19.59)$ & $<0.0001$ \\
\hline \multicolumn{5}{|l|}{ Single-vessel obstructive } \\
\hline CAD & $5.53(3.3-9.32)$ & $<0.0001$ & & \\
\hline Multivessel obstructive CAD & $6.22(3.6-10.7)$ & $<0.0001$ & & \\
\hline \multicolumn{5}{|l|}{ No. of segments with } \\
\hline Any CAD & $1.22(1.16-1.28)$ & $<0.0001$ & & \\
\hline Non-obstructive CAD & $1.18(1.12-1.24)$ & $<0.0001$ & & \\
\hline Obstructive CAD & $1.31(1.22-1.41)$ & $<0.0001$ & & \\
\hline \multicolumn{5}{|l|}{ Interaction term } \\
\hline Obstructive CAD $\times \mathrm{DM}$ & $6.71(4.0-11.3)$ & $<0.0001$ & & \\
\hline
\end{tabular}

overall population adjusting for other cardiovascular risk factors, although it approached significance on univariate analysis. However, the presence of significant stenosis was a strong independent predictor of vascular events with an odds ratio of 8.9 (4.0-19.59), $p<0.001$. Also, an interaction term obstructive $\mathrm{CAD} \times$ diabetes resulted in non-significance indicating that only the presence of significant CAD, but not that of diabetes, affected vascular events. Therefore, although cardiac risk was higher in the sample of diabetic patients than in non-diabetic patients, diabetes per se was not a CAD risk equivalent. This may reflect the greater importance of MSCT-CA evidence of obstructive CAD as well as symptomatic status for the prediction of cardiac events $[35,36]$. Additionally both DM and non-DM patients with non-obstructive $\mathrm{CAD}$ showed a higher event rate than patients with normal coronary arteries. It is known that almost two thirds of acute coronary syndromes are attributable to non-obstructive lesions $(<50 \%)$ owing to plaque disruption with superimposed thrombosis, whereas only $14 \%$ are attributable to a critical stenosis ( $>70 \%$ ) [37]. Importantly, our patients without CAD had a $100 \%$ event-free survival at mid-term follow-up. Therefore, since ideal risk stratification should identify patients who do not require further intervention, MSCT-CA may possess this attribute.

Finally, we demonstrated that coronary plaque score showed superior outcome classification ability when compared with the pre-test likelihood prediction model. Moreover, MSCT-CA variables provided significant incremental prognostic value over calcium score. This result is comparable to a recent study that demonstrated that MSCT$\mathrm{CA}$ provides additional information to calcium score regarding stenosis severity and plaque composition [38]. 
Table 5 Univariate and multivariate predictors of total cardiac events in diabetics and non-diabetics

\begin{tabular}{|c|c|c|c|c|c|c|c|c|}
\hline & \multicolumn{4}{|l|}{ Diabetics $(n=210)$} & \multicolumn{4}{|c|}{ Non-diabetics $(n=203)$} \\
\hline & \multicolumn{2}{|l|}{ Univariate analysis } & \multicolumn{2}{|c|}{ Multivariate analysis } & \multicolumn{2}{|l|}{ Univariate analysis } & \multicolumn{2}{|c|}{ Multivariate analysis } \\
\hline & $\mathrm{HR}(95 \% \mathrm{CI})$ & $p$ Value & HR $(95 \% \mathrm{CI})$ & $p$ Value & HR $(95 \% \mathrm{CI})$ & $p$ Value & HR $(95 \% \mathrm{CI})$ & $p$ Value \\
\hline \multicolumn{9}{|l|}{ Clinical characteristics } \\
\hline Age ( $>65$ years) & $3.80(1.94-7.44)$ & $<0.001$ & $2.22(1.09-4.51)$ & 0.03 & $1.97(0.81-4.80)$ & 0.14 & & \\
\hline Male gender & $0.89(0.46-1.73)$ & 0.74 & & & $3.41(1.0-11.58)$ & 0.05 & & \\
\hline Hypertension & $2.53(1.06-6.03)$ & 0.04 & & & $1.39(0.54-3.59)$ & 0.50 & & \\
\hline Family history & $1.54(0.80-2.95)$ & 0.19 & & & $1.76(0.72-4.28)$ & 0.22 & & \\
\hline Smoking & $2.03(1.07-3.87)$ & 0.03 & & & $1.87(0.78-4.49)$ & 0.16 & & \\
\hline Hypercholesterolaemia & $1.79(0.93-3.45)$ & 0.08 & & & $1.85(0.74-4.61)$ & 0.19 & & \\
\hline Obesity & $0.91(0.47-1.79)$ & 0.79 & & & $0.45(0.10-1.91)$ & 0.28 & & \\
\hline \multicolumn{9}{|l|}{ Symptoms } \\
\hline Asymptomatic & $0.34(0.14-0.81)$ & 0.016 & & & $0.28(0.06-1.19)$ & 0.09 & & \\
\hline Typical angina pectoris & $3.29(1.63-6.65)$ & $<0.001$ & $2.91(1.34-6.31)$ & 0.007 & $9.47(3.93-22.8)$ & $<0.0001$ & $2.75(1.06-7.1)$ & 0.03 \\
\hline Atypical angina pectoris & $0.63(0.30-1.29)$ & 0.21 & & & $0.39(0.13-1.17)$ & 0.09 & & \\
\hline Dyspnoea & $2.05(0.99-4.24)$ & 0.05 & $3.8(1.7-8.51)$ & 0.001 & $0.83(0.28-2.48)$ & 0.74 & & \\
\hline \multicolumn{9}{|l|}{ MSCT characteristics } \\
\hline $\mathrm{CACS}>100$ & $8.68(3.82-19.7)$ & $<0.0001$ & & & $16.4(4.86-55.9)$ & $<0.0001$ & $5.57(1.44-21.5)$ & 0.01 \\
\hline $\mathrm{CACS}>400$ & $6.46(3.33-12.54)$ & $<0.0001$ & & & $4.65(1.86-11.61)$ & 0.001 & & \\
\hline Absence of plaques & $0.03(0.001-0.5)$ & 0.02 & & & $0.02(0.001-0.93)$ & 0.04 & & \\
\hline Non-obstructive CAD & $0.30(0.13-0.73)$ & 0.01 & & & $0.29(0.09-0.85)$ & 0.02 & & \\
\hline Obstructive CAD & $12.19(5.10-29.13)$ & $<0.0001$ & $6.38(2.33-17.5)$ & $<0.001$ & $23.7(7.89-70.9)$ & $<0.0001$ & $7.43(2.1-26.7)$ & 0.002 \\
\hline Single-vessel obstructive CAD & $4.04(2.12-7.71)$ & $<0.0001$ & & & $8.12(3.39-19.46)$ & $<0.0001$ & & \\
\hline Multivessel obstructive CAD & $4.25(2.19-8.25)$ & $<0.0001$ & & & $11.46(4.38-29.9)$ & $<0.0001$ & & \\
\hline \multicolumn{9}{|l|}{ No. of segments with } \\
\hline Any CAD & $1.22(1.14-1.3)$ & $<0.0001$ & $1.1(1.001-1.2)$ & 0.04 & $1.21(1.11-1.31)$ & $<0.0001$ & & \\
\hline Non-obstructive CAD & $1.18(1.10-1.25)$ & $<0.0001$ & & & $1.17(1.07-1.28)$ & 0.001 & & \\
\hline Obstructive CAD & $1.24(1.14-1.36)$ & $<0.0001$ & & & $2.1(1.6-2.6)$ & $<0.0001$ & & \\
\hline
\end{tabular}

$\mathrm{CAD}=$ coronary artery disease $\mathrm{HR}=$ hazard ratio $\mathrm{CI}=$ confidence interval

This additional information was shown to translate into incremental value for risk stratification.

In our study, both DM and non-DM patients with typical angina were at higher risk of adverse outcomes than patients among the other clinically relevant categories. Our results are similar to those reported in previous prognostic studies in patients undergoing exercise testing $[39,40]$. Interestingly, in our study we registered two cardiac deaths among asymptomatic DM patients with obstructive CAD on MSCT-CA. Moreover, these patients showed more than three times the prevalence of obstructive CAD among asymptomatic non-DM patients. It is known that ischaemic chest pain is blunted in DM. Myocardial ischaemia or myocardial infarction may be associated with only mild symptoms or may be totally silent owing to autonomic neuropathy. Silent ischaemia, in particular, is a concern in about $20 \%$ of DM patients [41, 42].

In our study dyspnoea has emerged a strong prognostic indicator among DM patients. Although dyspnoea is the most common complaint of patients with cardiopulmonary diseases, there has been only limited investigation of its prognostic significance among patients referred for cardiac evaluation. A previous study conducted with myocardial perfusion single-photon emission computed tomography (SPECT) demonstrated that DM patients with shortness of breath and no history of CAD had a significantly worse outcome and a higher likelihood of ischaemic abnormalities than those who had typical angina pectoris or who were asymptomatic [43]. Furthermore, another study involving a large cohort of patients undergoing SPECT showed that among patients with no known history of CAD, those with dyspnoea had four times the risk of sudden death from cardiac causes of asymptomatic patients and more than twice the risk of patients with typical angina [44]. In this prognostic study, interestingly, patients with dyspnoea had a significantly higher rate of diabetes than those in other clinical subgroups.

These findings complement the results of two earlier studies that investigated the prognostic role of dyspnoea in 

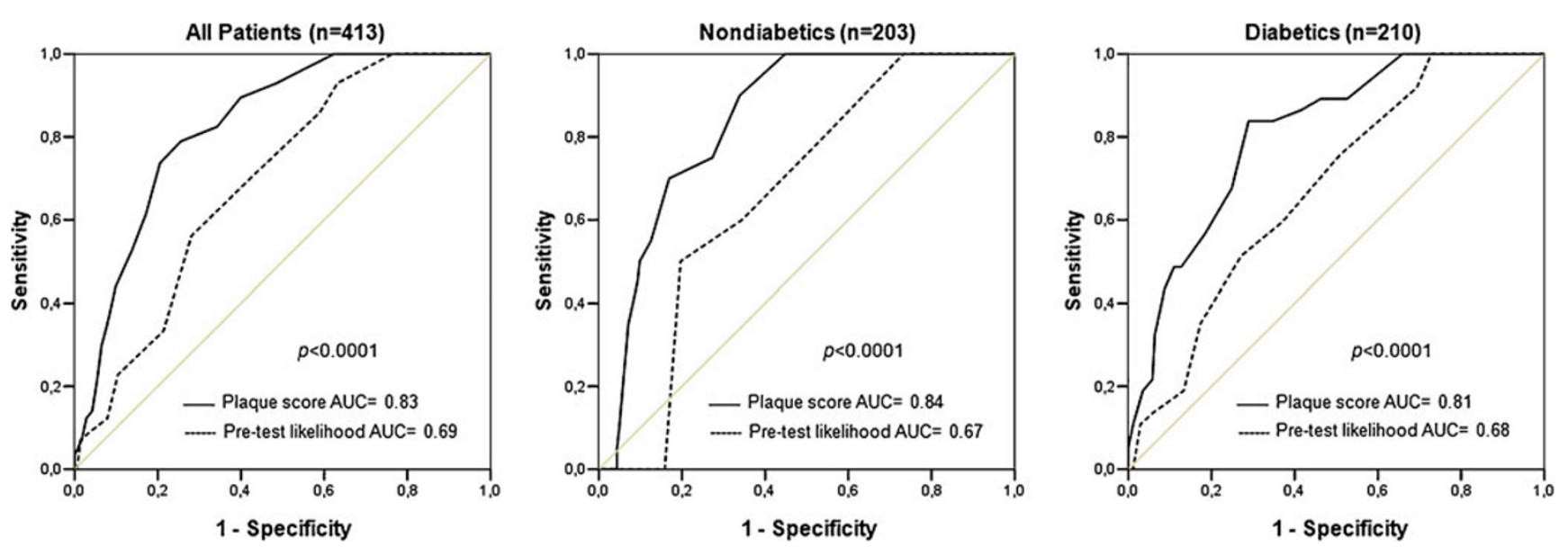

Fig. 5 Receiver-operating characteristic curve analysis demonstrating the incremental ability of total plaque score over pre-test likelihood in predicting MACE in all patients, non-diabetics and diabetics. AUC, area under the curve; MACE, major adverse cardiac events

patients undergoing stress echocardiography or exercise stress testing $[45,46]$. One potential explanation is that, even in the absence of chest pain, the presence of symptoms such as dyspnoea may serve as an angina equivalent or a marker of underlying cardiac disease [47].

\section{Study limitations}

This study has several potential limitations. First, there was the enrolment bias that led to such a high proportion of diabetics.

Second, complete information concerning the degree of metabolic control of DM and the status of secondary organ involvement and of autonomic dysfunction was missing.
This limitation was mainly due to the exploratory nature of the study, which was aimed at investigating the prognostic role of MSCT-CA and symptoms on outcome in "real world" DM patients without known CAD rather than on the degree of diabetes control.

Another limitation of the study is that we included in the multivariate analysis patients who underwent early revascularization procedures ( $<60$ days after the examination) that are generally performed as a direct consequence of the MSCT-CA findings, such as evidence of obstructive CAD. Conversely, decisions to perform late revascularization procedures ( $\geq 60$ days after MSCT-CA examination) are usually not significantly influenced by the results of the examination, but instead by worsening clinical status, such

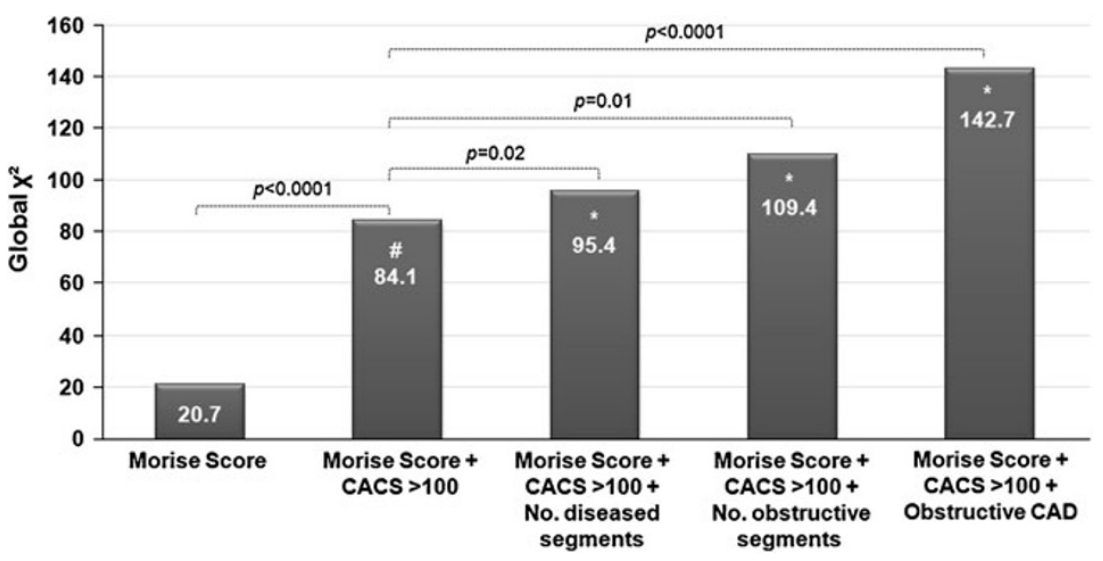

Fig. 6 Bar graphs illustrating the incremental prognostic value (depicted by global $\chi^{2}$ value on the $y$-axis) of plaque burden on MSCT-CA (defined as the number of diseased segments or number of segments with obstructive CAD) and obstructive CAD ( $>50 \%$ stenosis) on MSCT-CA over clinical baseline (Morise score) and CACS. CACS has a significant incremental prognostic value over
Morise score (\#). A further incremental prognostic value over Morise score and CACS was observed with the addition of MSCT-CA $(*)$. $\mathrm{CAD}=$ coronary artery disease; MSCT-CA $=$ multislice computed tomography coronary angiography; CACS, coronary artery calcium score 
that late revascularizations represent a surrogate for disease progression. However, it is worthwhile noting that all decisions regarding revascularization were based on symptoms and/or the presence of concomitant ischaemia on noninvasive testing, rather than on the arbitrary evidence of obstructive CAD on MSCT-CA. Furthermore, in our study, a parallel survival model for MACE excluding early revascularizations demonstrated that DM patients remained associated with worse outcome; moreover, the presence of obstructive CAD and typical angina remained associated with outcome in both DM and non-DM patients. Therefore, our results do not seem to be affected by treatment bias.

Another shortcoming could be that men and women may report symptoms differently; therefore, the risk of cardiovascular events may be affected by gender differences among symptom categories.

Furthermore, the reproducibility of the self-reported information about presenting symptoms was not evaluated. Pulmonary and cardiac diseases are responsible for almost all cases of dyspnoea, and historical or functional testing information regarding lung disorders was not systematically obtained. A systematic analysis of the left ventricular ejection fraction or exercise-induced dynamic functional mitral regurgitation as a mechanism for inducing dyspnoea was not performed. Moreover, anaemia and psychogenic causes of dyspnoea were not evaluated.

As patients were categorised as having dyspnoea if they had no history of concomitant chest discomfort, we were unable to evaluate the potential interaction between dyspnoea and the presence of chest pain.

Finally, both the relatively small number of patients enrolled and the duration of follow-up in the present study are other important limitations. Larger studies or registry data are required to definitively address this issue. On the basis of these considerations, caution should be used in making definitive inferences from our results.

\section{Conclusion}

In this study MSCT-CA evidence of obstructive CAD provided incremental information for predicting future cardiac events in addition to standard risk factor assessment and presenting symptom characteristics. MSCT-CA with no evidence of obstructive or non-obstructive CAD was not associated with any cardiac events during the study period in both DM and non-DM patients.

The study also demonstrated that dyspnoea is another important symptom in the assessment of prognosis among diabetic patients.

In standard assessment, MSCT-CA may give valuable prognostic information for the evaluation of patients with suspected CAD, particularly those at low or intermediate risk of having significant CAD.

\section{Conflicts of interest None.}

Open Access This article is distributed under the terms of the Creative Commons Attribution Noncommercial License which permits any noncommercial use, distribution, and reproduction in any medium, provided the original author(s) and source are credited.

\section{References}

1. Wild S, Roglic G, Green A, Sicree R, King H (2004) Global prevalence of diabetes: estimates for the year 2000 and projections for 2030. Diab Care 27:1047-1053

2. Rydén L, Standl E, Bartnik M, Van den Berghe G, Betteridge J, de Boer MJ et al (2007) Guidelines on diabetes, pre-diabetes, and cardiovascular diseases: executive summary. The Task Force on Diabetes and Cardiovascular Diseases of the European Society of Cardiology (ESC) and of the European Association for the Study of Diabetes (EASD). Eur Heart J 28:88-136

3. Nathan DM, Meigs J, Singer DE (1997) The epidemiology of cardiovascular disease in type 2 diabetes mellitus: how sweet it is...or is it? Lancet 350(Suppl 1):4-9

4. Goraya TY, Leibson CL, Palumbo PJ, Weston SA, Killian JM, Pfeifer EA et al (2002) Coronary atherosclerosis in diabetes mellitus: a population-based autopsy study. J Am Coll Cardiol 40:946-953

5. Scognamiglio R, Negut C, Ramondo A, Tiengo A, Avogaro A (2006) Detection of coronary artery disease in asymptomatic patients with type 2 diabetes mellitus. J Am Coll Cardiol 47:6571

6. Hachamovitch R, Hayes SW, Friedman JD, Cohen I, Berman DS (2003) Comparison of the short-term survival benefit associated with revascularization compared with medical therapy in patients with no prior coronary artery disease undergoing stress myocardial perfusion single photon emission computed tomography. Circulation 107:2900-2907

7. Sorajja P, Chareonthaitawee P, Rajagopalan N, Miller TD, Frye RL, Hodge DO et al (2005) Improved survival in asymptomatic diabetic patients with high-risk SPECT imaging treated with coronary artery bypass grafting. Circulation 112:1311-1316

8. Kamalesh M, Feigenbaum H, Sawada S (2007) Assessing prognosis in patients with diabetes mellitus - the Achilles' heel of cardiac stress imaging tests? Am J Cardiol 99:1016-1019

9. Diamond GA (1983) A clinically relevant classification of chest discomfort. J Am Coll Cardiol 1:574-575

10. Pryor DB, Shaw L, McCants CB, Lee KL, Mark DB, Harrell FE $\mathrm{Jr}$ et al (1993) Value of the history and physical in identifying patients at increased risk for coronary artery disease. Ann Intern Med 118:81-90

11. Constant J (1983) The clinical diagnosis of nonanginal chest pain: the differentiation of angina from nonanginal chest pain by history. Clin Cardiol 6:11-16

12. Nesto RW (1999) Screening for asymptomatic coronary artery disease in diabetes. Diab Care 22:1393-1395

13. Hendel RC, Patel MR, Kramer CM, Poon M, Hendel RC, Carr JC et al (2006) ACCF/ACR/SCCT/SCMR/ASNC/NASCI/SCAI/SIR 2006 appropriateness criteria for cardiac computed tomography and cardiac magnetic resonance imaging: a report of the American College of Cardiology Foundation Quality Strategic Directions 
Committee Appropriateness Criteria Working Group, American College of Radiology, Society of Cardiovascular Computed Tomography, Society for Cardiovascular Magnetic Resonance, American Society of Nuclear Cardiology, North American Society for Cardiac Imaging, Society for Cardiovascular Angiography and Interventions, and Society of Interventional Radiology. J Am Coll Cardiol 48:1475-1497

14. Schroeder S, Achenbach S, Bengel F, Burgstahler C, Cademartiri F, de Feyter P et al (2008) Cardiac computed tomography: indications, applications, limitations, and training requirements: report of a Writing Group deployed by the Working Group Nuclear Cardiology and Cardiac CT of the European Society of Cardiology and the European Council of Nuclear Cardiology. Eur Heart J 29:531-556

15. Maffei E, Palumbo A, Martini C, Meijboom W, Tedeschi C, Spagnolo P et al (2010) Diagnostic accuracy of 64-slice computed tomography coronary angiography in a large population of patients without revascularization: registry data and review of multicentre trials. Radiol Med 115:368-384

16. Pundziute G, Schuijf JD, Jukema JW, Boersma E, de Roos A, van der Wall EE et al (2007) Prognostic value of multislice computed tomography coronary angiography in patients with known or suspected coronary artery disease. J Am Coll Cardiol 49:62-70

17. Min JK, Shaw LJ, Devereux RB, Okin PM, Weinsaft JW, Russo DJ et al (2007) Prognostic value of multidetector coronary computed tomographic angiography for prediction of all-cause mortality. J Am Coll Cardiol 50:1161-1170

18. Gaemperli O, Valenta I, Schepis T, Husmann L, Scheffel H, Desbiolles L et al (2008) Coronary 64-slice CT angiography predicts outcome in patients with known or suspected coronary artery disease. Eur Radiol 18:1162-1173

19. Van Werkhoven JM, Cademartiri F, Seitun S, Maffei E, Palumbo A, Martini C et al (2010) Diabetes: prognostic value of CT coronary angiography-comparison with a nondiabetic population. Radiology 256:83-92

20. Carrigan TP, Nair D, Schoenhagen P, Curtin RJ, Popovic ZB, Halliburton S et al (2009) Prognostic utility of 64-slice computed tomography in patients with suspected but no documented coronary artery disease. Eur Heart J 30:362-371

21. Hadamitzky M, Freissmuth B, Meyer T, Hein F, Kastrati A, Martinoff S et al (2009) Prognostic value of coronary computed tomographic angiography for prediction of cardiac events in patients with suspected coronary artery disease. JACC Cardiovasc Imaging 2:404-411

22. Chow BJ, Wells GA, Chen L, Yam Y, Galiwango P, Abraham A et al (2010) Prognostic value of 64-slice cardiac computed tomography severity of coronary artery disease, coronary atherosclerosis, and left ventricular ejection fraction. J Am Coll Cardiol 55:1017-1028

23. Report of the Expert Committee on the Diagnosis and Classification of Diabetes Mellitus (2003) Diabetes Care 26(Suppl 1):5-20

24. 2003 European Society of Hypertension-European Society of Cardiology guidelines for the management of arterial hypertension (2003) J Hypertens 21:1011-1053

25. Executive Summary of the Third Report of The National Cholesterol Education Program (NCEP) Expert Panel on Detection, Evaluation, and Treatment of High Blood Cholesterol in Adults (Adult Treatment Panel III) (2001) JAMA 285:2486-2497

26. Clinical Guidelines on the Identification, Evaluation, and Treatment of Overweight and Obesity in Adults - the Evidence Report. National Institutes of Health (1998) Obes Res 6(Suppl 2):51-209

27. Taylor AJ, Bindeman J, Feuerstein I, Cao F, Brazaitis M, O’Malley PG (2005) Coronary calcium independently predicts incident premature coronary heart disease over measured cardiovascular risk factors: mean three-year outcomes in the Prospective Army Coronary Calcium (PACC) project. J Am Coll Cardiol 46:807-814

28. Morise AP, Haddad WJ, Beckner D (1997) Development and validation of a clinical score to estimate the probability of coronary artery disease in men and women presenting with suspected coronary disease. Am J Med 102:350-356

29. Agatston AS, Janowitz WR, Hildner FJ, Zusmer NR, Viamonte M Jr, Detrano R (1990) Quantification of coronary artery calcium using ultrafast computed tomography. J Am Coll Cardiol 15:827-832

30. Austen WG, Edwards JE, Frye RL, Gensini GG, Gott VL, Griffith LS et al (1975) A reporting system on patients evaluated for coronary artery disease. Report of the Ad Hoc Committee for grading of Coronary Artery Disease, Council on Cardiovascular Surgery, American Heart Association. Circulation 51:5-40

31. Achenbach S, Moselewski F, Ropers D, Ferencik M, Hoffmann U, MacNeill B et al (2004) Detection of calcified and noncalcified coronary atherosclerotic plaque by contrast-enhanced, submillimeter multidetector spiral computed tomography: a segment-based comparison with intravascular ultrasound. Circulation 109:14-17

32. Myocardial infarction redefined - a consensus document of The Joint European Society of Cardiology/American College of Cardiology Committee for the redefinition of myocardial infarction (2000) J Am Coll Cardiol 36:959-969

33. Wilson PW, D'Agostino RB, Levy D, Belanger AM, Silbershatz H, Kannel WB (1998) Prediction of coronary heart disease using risk factor categories. Circulation 97:1837-1847

34. Diamond GA, Forrester JS (1979) Analysis of probability as an aid in the clinical diagnosis of coronary-artery disease. $\mathrm{N}$ Engl $\mathrm{J}$ Med 300:1350-1358

35. Alderman EL, Corley SD, Fisher LD, Chaitman BR, Faxon DP, Foster ED et al (1993) Five-year angiographic follow-up of factors associated with progression of coronary artery disease in the Coronary Artery Surgery Study (CASS). CASS Participating Investigators and Staff. J Am Coll Cardiol 22:11411154

36. Giroud D, Li JM, Urban P, Meier B, Rutishauer W (1992) Relation of the site of acute myocardial infarction to the most severe coronary arterial stenosis at prior angiography. Am J Cardiol 69:729-732

37. Falk E, Shah PK, Fuster V (1995) Coronary plaque disruption. Circulation 92:657-671

38. Van Werkhoven JM, Schuijf JD, Gaemperli O, Jukema JW, Kroft LJ, Boersma E et al (2009) Incremental prognostic value of multislice computed tomography coronary angiography over coronary artery calcium scoring in patients with suspected coronary artery disease. Eur Heart J 30:2622-2629

39. Christopher JR, Pothier CE, Blackstone EH, Lauer MS (2004) Prognostic importance of presenting symptoms in patients undergoing exercise testing for evaluation of known or suspected coronary disease. Am J Med 117:380-389

40. Sekhri N, Feder GS, Junghans C, Eldridge S, Umaipalan A, Madhu $\mathrm{R}$ et al (2008) Incremental prognostic value of the exercise electrocardiogram in the initial assessment of patients with suspected angina: cohort study. BMJ 337:2240

41. Wackers FJ, Young LH, Inzucchi SE, Chyun DA, Davey JA, Barrett EJ et al (2004) Detection of silent myocardial ischemia in asymptomatic diabetic subjects: the DIAD study. Diab Care 27:1954-1961, Erratum in: (2005) Diabetes Care 28:504

42. Zellweger MJ, Hachamovitch R, Kang X, Hayes SW, Friedman JD, Germano G et al (2009) Threshold, incidence, and predictors of prognostically high-risk silent ischemia in asymptomatic patients without prior diagnosis of coronary artery disease. J Nucl Cardiol 16:193-200

43. Zellweger MJ, Hachamovitch R, Kang X, Hayes SW, Friedman JD, Germano G et al (2004) Prognostic relevance of symptoms 
versus objective evidence of coronary artery disease in diabetic patients. Eur Heart J 25:543-550

44. Abidov A, Rozanski A, Hachamovitch R, Hayes SW, AboulEnein F, Cohen I et al (2005) Prognostic Significance of Dyspnea in Patients Referred for Cardiac Stress Testing. N Engl J Med 353:1889-1898

45. Bergeron S, Ommen SR, Bailey KR, Oh JK, McCully RB, Pellikka PA (2004) Exercise echocardiographic findings and outcome of patients referred for evaluation of dyspnea. J Am Coll Cardiol 43:2242-2246

46. Christopher Jones R, Pothier CE, Blackstone EH, Lauer MS (2004) Prognostic importance of presenting symptoms in patients undergoing exercise testing for evaluation of known or suspected coronary disease. Am J Med 117:380-389

47. Marwick TH (2005) Dyspnea and risk in suspected coronary disease. N Engl J Med 353:1963-1965 